\title{
Generación de señales para sistemas radio sobre fibra basados en combinación óptica
}

\section{Signal Generation for Radio Over Fibre Systems Based on Optical Combination}

\author{
Muñoz-Ortiz Rafael Oswaldo \\ Universidad Distrital Francisco José de Caldas \\ Facultad de Ingeniería \\ Correo: romunozo@correo.udistrital.edu.co \\ Cely-Mancipe Manuel Arturo \\ Facultad de Ingeniería \\ Universidad Distrital Francisco José de Caldas \\ Correo:macelym@correo.udistrital.edu.co
}

\author{
Puerto-Leguizamón Gustavo Adolfo \\ Facultad de Ingeniería \\ Universidad Distrital Francisco José de Caldas \\ Correo:gapuerto@udistrital.edu.co \\ Suárez-Fajardo Carlos Arturo \\ Facultad de Ingeniería \\ Universidad Distrital Francisco José de Caldas \\ Correo: csuarezf@udistrital.edu.co
}

Información del artículo: recibido: octubre de 2014, aceptado: marzo de 2015

\section{Resumen}

Este artículo presenta un estudio comparativo de técnicas de generación de señales para el transporte de información en sistemas de Radio sobre Fibra (RoF) usando técnicas de combinación óptica. En estos sistemas las portadoras de radiofrecuencia se transportan como subportadoras de canal óptico sobre un enlace en conexiones punto-punto o punto-multipunto. Este estudio se centra en tres diferentes técnicas de generación: brazo doble, generación en paralelo y generación serial. Para cada una de ellas se define un modelo analítico con el fin de describir los elementos, la configuración y los procesos involucrados en la generación de señales RoF. Posteriormente se modelan las funciones de campo eléctrico obtenidas con el fin de evaluar la calidad de las señales generadas en función de los índices de modulación establecidos. Los resultados obtenidos muestran que los índices de modulación para los esquemas de brazo doble y serial presentan una fuerte dependencia entre sí, mientras la configuración de generación en paralelo permite una mayor flexibilidad en el establecimiento de los índices de modulación. Para cada esquema se obtiene el valor óptimo de índices de modulación a fin de garantizar una buena calidad para ambas señales a la salida del transmisor RoF.

\section{Descriptores:}

- fotónica de microondas

- índice de modulación

- modulador interferométrico

- modulación óptica

- radio sobre fibra 


\begin{abstract}
This paper presents a comparative study of the signal generation techniques for the transport of information in Radio over Fiber (RoF) systems by using optical combination techniques. In these systems, the radio frequency carriers are conveyed as optical subcarriers over a link in a point-to-point or point-to-multipoint connectivity paradigm. This study is focused on three different generation techniques: double arm, parallel and serial generation. An analytical model to describe the elements, the configuration and the processes involved in the RoF signal generation is defined for each one of them. Subsequently, a modeling of the obtained electrical field functions allows assessing the quality of the generated signals based on the modulation depth. Results show that while there is a trade-off between the modulation depths of both signals for the double arm and serial configuration, the parallel technique relaxes the establishment of different modulation depths. An optimum configuration that guarantees a good quality for the generated signals is found for each generation technique.
\end{abstract}

\section{Introducción}

La demanda de altas tasas de transmisión y gran ancho de banda en redes fijas e inalámbricas se ha incrementado en los últimos años y se pronostica un comportamiento similar en los años venideros. El fabricante de equipos de telecomunicaciones Cisco Systems publicó un reporte que pronostica el tráfico de datos a nivel mundial hasta el año 2018, con base en mediciones del tráfico actual de Internet (Cisco, 2014). El reporte especifica que el tráfico IP global anual superará el umbral del zettabyte alcanzando los 1.4 zettabytes a finales de 2017. A 2014, el tráfico IP global experimentó un aumento de cuatro veces el valor con respecto al presentado en 2009, asimismo, se espera que el tráfico actual se triplique en los próximos 5 años. En general, el tráfico IP crecerá a una tasa anual compuesta de $23 \%$ desde 2012 hasta 2017. El tráfico en el segmento metropolitano superará el de la red de transporte en 2014 y representará 58\% del tráfico IP total en 2017. Entre 2012 y 2017 en este segmento de red, el tráfico crecerá casi dos veces más que el tráfico de la red troncal. Del mismo modo se pronostica que para 2017 casi la mitad de todo el tráfico IP se originará en dispositivos que no son computadores personales. En 2012, solo 26\% del tráfico IP de consumo se originó en dispositivos no-PC, pero para 2017 la proporción de tráfico IP no-PC crecerá hasta $49 \%$. El tráfico originado en computadores personales crecerá a una tasa compuesta anual de $14 \%$, mientras que el tráfico originado en tablets, teléfonos móviles, televisores y dispositivos de comunicación máquinamáquina (M2M) tendrán tasas de crecimiento del tráfico generado de $104 \%, 79 \%$, $24 \%$ y $82 \%$, respectivamente. El tráfico de los dispositivos inalámbricos y móviles superará el de dispositivos cableados en 2016. En 2017, los dispositivos conectados por cable representarán $45 \%$ del tráfico IP, mientras que WiFi y dispositivos móviles representarán $55 \%$ del tráfico IP. Así, a corto plazo se prevé un gran aumento del ancho de banda originado desde dispositivos móviles.

Hoy en día las redes de acceso ópticas pueden proporcionar gran ancho de banda a usuarios fijos. Por otro lado, las redes inalámbricas ofrecen una movilidad deseable a los usuarios, pero no cumplen con los requerimientos de ancho de banda. Además, tener redes de acceso separadas genera un alto costo en términos de operación y mantenimiento. Todo esto sugiere la integración de dichas redes en una sola infraestructura compartida para la futura distribución de contenidos a usuarios fijos y móviles. En este contexto, los sistemas de radio sobre fibra (RoF) y fibra hasta el hogar (FTTH) son dos candidatos serios para consolidarse en el segmento de redes de acceso inalámbrico y fijo, respectivamente, debido al gran ancho de banda que soportan. Aunque los sistemas RoF se pueden implementar en redes troncales cubriendo largas distancias (Marciniak, 2004), el mayor punto de atracción radica en el despliegue de sistemas RoF en redes de acceso inalámbrico de banda ancha que permita el transporte y la distribución de las portadoras de RF de cualquier red inalámbrica utilizando una infraestructura de red FTTH. Mientras que la atenuación en espacio libre de frecuencias en la banda de microondas puede llegar a ser mayor de $10 \mathrm{~dB} / \mathrm{km}$, en fibra óptica estándar monomodo, la atenuación es de $0.2 \mathrm{~dB} / \mathrm{km}$ independientemente de la banda de frecuencia que se transporte. Este hecho sumado al gran ancho de banda ofrecido por la fibra óptica, constituyen las principales razones por las cuales son atractivas las tec- 
nologías de transmisión RoF. En este escenario la principal preocupación y a la vez el desafío, se traduce en cómo transmitir las señales de banda base de las redes FTTH y de radiofrecuencia (RF) de las redes inalámbricas en una sola longitud de onda sobre una sola fibra de una manera costo-efectiva y con una calidad aceptable.

A la fecha se han realizado diferentes trabajos que demuestran las ventajas de implementar sistemas de transporte de señales de radiofrecuencia sobre enlaces de fibra óptica en diferentes escenarios y aplicaciones. Zhensheng et al. (2007) discuten las tecnologías habilitantes que permiten el desarrollo de sistemas RoF que incluye esquemas para generación óptica de ondas milimétricas y elevadores de frecuencia, Gee-Kung et al. (2007) presenta varias técnicas para la implementación de redes de acceso ópticas-inalámbricas basadas en procesos de modulación externa realizada en un modulador tipo Mach-Zehnder (MZ). Por otro lado, la modulación simultánea de señales en banda base y RF en un modulador óptico la demostró experimentalmente Chun-Ting et al. (2007). En el contexto de estudios sobre las limitaciones en las prestaciones y desempeño en la transmisión de señales analógicas en enlaces de fibra, Castleford et al. (2001) presentaron el efecto de intermodulaciones en banda y fuera de banda de canales multiplexados en longitud de onda (WDM) en una red de transporte RoF. Asimismo, se han reportado estudios sobre los efectos de las características no-lineales de diodos láser configurados en modulación directa (Mizuguti et al., 1993) y efectos de distorsión causados por moduladores tipo MZ en enlaces RoF (Way,1993; Cox,1993). Posteriormente en Ackerman et al. (1993); Sabido y Kazovsky (2001) se definieron parámetros para identificar el máximo rango dinámico y las mínimas pérdidas de inserción en un enlace óptico modulado externamente con subportadoras de RF y en Sabella (2001) se ampliaron los estudios mencionados anteriormente con el reporte y demostración de un análisis de prestaciones de calidad de un sistema de transmisión RoF considerando requerimientos generados por efectos de propagación de ondas milimétricas además de la evaluación de diferentes formatos de modulación. Asimismo, Kurniawan et al. (2006) presentan un estudio sobre los efectos de ruido e intermodulación en enlaces RoF que permiten identificar las limitaciones en el desempeño del sistema, así como un análisis de la relación portadora-banda lateral en Lim et al. (2006) y diferentes estrategias para reducir la figura de ruido en enlaces de fibra óptica modulados con señales analógicas (Karim y Devenport, 2007). En este contexto, un análisis sobre la optimización del punto de cuadratura de un modulador óptico tipo MZ para aplicaciones RoF se presentó en Zongjie et al. (2011). Recientemente se publicó un estudio sobre el análisis de moduladores ópticos tipo MZ y de electro-absorción para aplicaciones de RoF y comunicaciones ópticas de espacio libre (Prabu et al., 2012).

En este artículo se presenta un estudio comparativo de calidad de señal en el cual la función de campo obtenida a la salida de un modulador tipo MZ se modela para diferentes configuraciones de generación de señales RoF en función de la variación de los índices de modulación de las señales que alimentan el modulador óptico.

\section{Modulación electro-óptica}

Los mecanismos de modulación óptica se basan en alterar alguno de los parámetros de una señal óptica de forma proporcional a una segunda señal eléctrica. El efecto de la señal moduladora sobre la señal modulada se evalúa de forma cuantitativa mediante el índice de modulación, cuanto mayor es dicho índice, mayor es la variación del parámetro modificado en la señal portadora para la misma señal moduladora. La señal óptica generada por un láser se puede modular de forma directa mediante la variación de la corriente inyectada al dispositivo, o externamente, haciendo pasar el haz de luz emitido a través de un elemento controlable que cambia la amplitud y la fase de la luz. En este contexto, se pueden considerar láseres directamente modulados y láseres modulados externamente. El presente estudio está basado en el paradigma de modulación externa en moduladores tipo Mach-Zehnder (MZ), los cuales se basan en el efecto electroóptico lineal o efecto Pockels (Cho et al., 1996). Un modulador tipo MZ utiliza una estructura interferométrica implementada sobre un sustrato de Niobato de Litio (LiNbO3), como se muestra en la figura 1. En este dispositivo, la luz se divide en partes iguales entre dos guías de ondas paralelas en la superficie del sustrato y se recombina nuevamente en la salida. La variación en el índice de refracción se traduce en un cambio de alguna de las propiedades de la señal óptica: amplitud, frecuencia, fase o polarización, de-

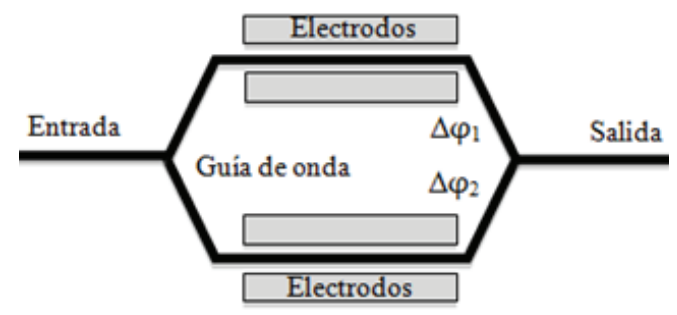

Figura 1. Modulador MZ de control doble 
pende de la configuración del dispositivo, en particular, de las corrientes de polarización que controlan el elemento modulador y que tienen incidencia directa en los índices de modulación, relación de extinción y potencia de la señal modulada.

En este contexto, una tensión de control (V) aplicada a los electrodos que se muestran en la figura 1 , genera un cambio en el índice de refracción a través del efecto electroóptico. Esto provoca un cambio en la constante de propagación y un desplazamiento de fase relativo en el punto donde las dos ondas se combinan. Cuando no hay tensión, el desplazamiento de fase relativa es cero y la señal recombinada sale del dispositivo sin atenuación (a excepción de las pérdidas en las guías de ondas). Cuando se aplica una tensión $(V \pi)$ que produce un desplazamiento de fase de $\pi$ entre los dos brazos, la señal se extingue, por lo tanto, el dispositivo actúa como un interruptor controlado por tensión. Estos dispositivos funcionan a velocidades de hasta $50 \mathrm{~Gb} / \mathrm{s}$ (Yamada et al., 2011). Es importante destacar que $V \pi$ es un parámetro propio de cada modulador.

Un modulador tipo MZ también permite la aplicación de voltajes de control en ambas guías de onda, lo que resulta en un dispositivo modulador externo de doble control. Esta característica permite la generación de señales en cuadratura arbitrarias (Ho, 2005) que encuentran aplicación en procedimientos de transmisión, tales como modulación de portadora óptica suprimida. Los moduladores externos también se pueden utilizar para la modulación de fase en sistemas coherentes usando una única guía de onda con un índice de refracción modulado electroópticamente. De esta manera, la modulación externa ofrece ventajas sobre la modulación directa del diodo láser en sistemas de fibra óptica, principalmente en términos de ancho de banda y rangos de linealidad. Este aspecto impulsó el desarrollo de tecnologías de fabricación de los moduladores ópticos integrados a un nivel actual de madurez muy alto (Kikuchi et al., 2012; Kaiser et al., 2012). La figura 2 muestra la curva de transferencia de un modulador tipo MZ. Esta curva representa la transferencia de potencia óptica del dispositivo, en función del desfase electroóptico inducido sobre la señal óptica.

Este desfase depende a su vez de la tensión de polarización aplicada sobre los electrodos. Como se puede observar, existe una región donde la función de transferencia tiene carácter lineal, y por consiguiente, resulta óptima para la modulación de la señal eléctrica sobre la portadora óptica. El dispositivo trabaja en régimen lineal cuando se aplica una tensión de polarización tal que se induce un desfase sobre la señal óptica igual a $\pi / 2$, y además los niveles de tensión aplicados son lo suficientemente pequeños para no distorsionar la señal de información. Las ecuaciones de modelado del modulador MZ tienen como finalidad obtener la expresión del campo eléctrico de la señal óptica a la salida del dispositivo, en función de las diferentes señales de entrada y de algunos parámetros del mismo. Como punto de partida, se formula una primera aproximación que proporciona el campo eléctrico a la salida del dispositivo en función del campo eléctrico a la entrada y de los desfases inducidos por las señales eléctricas aplicadas sobre los electrodos del modulador MZ como consecuencia del efecto electroóptico. La ecuación de campo se establece a partir de la geometría del modulador. En el dispositivo mostrado en la figura 1 , se aplica una señal eléctrica sobre uno de los dos brazos del interferómetro. Esta señal provoca mediante el efecto electroóptico un cambio de fase sobre la señal óptica que se propaga por dicho brazo (Agrawal, 2002). La función de transferencia se puede expresar en términos del coeficiente de acoplo de la propagación del campo y el desfase producido en la señal en ambas ramas del interferómetro de la siguiente forma (Kurniawan, 2006):

$E_{\text {out }}(t)=\left(\frac{1}{\sqrt{2}}\right) E_{\text {in }}(t) \frac{\left[A_{1} e^{\left(j \Delta \varphi_{1}\right)}+A_{2} e^{\left(j \Delta \varphi_{2}\right)}\right]}{\alpha}$

donde:

$\alpha \quad=$ atenuación de la señal a su paso por el dispositivo

$\mathrm{A}_{1} \mathrm{y} \mathrm{A}_{2}=$ coeficientes de acoplo de las ramas superior e inferior, respectivamente, en el modelo del modulador de control doble mostrado en la figura 1

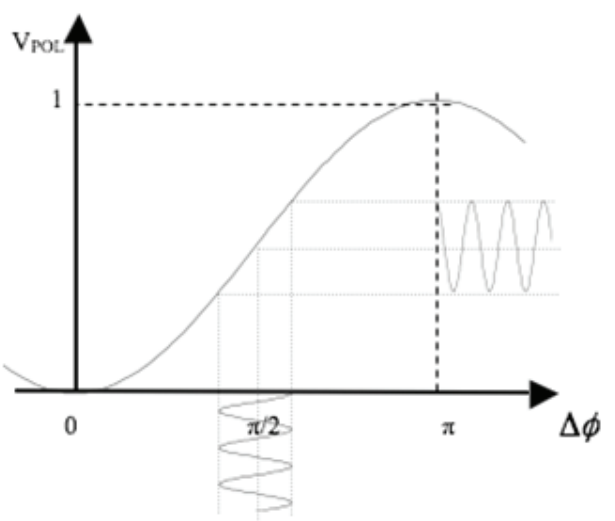

Figura 2. Curva de transferencia de potencia de un modulador $\mathrm{MZ}$ 
$\phi_{1}, \phi_{2}=$ desfases en cada una de las ramas debido al efecto electroóptico. Estas variables se pueden expresar de la siguiente forma

$A_{1}=a=\sqrt{0.5+\varepsilon}$

$A_{2}=\sqrt{1-\alpha^{2}}$

$\Delta \varphi_{1}=\frac{\pi}{V_{\pi(R F)}} V_{1(R F)}(t)+\frac{\pi}{V_{\pi(d c)}} V_{1(d c)}$

$\Delta \varphi_{2}=\frac{\pi}{V_{\pi(R F)}} V_{2(R F)}(t)+\frac{\pi}{V_{\pi(d c)}} V_{2(d c)}$

El término $\varepsilon$ en (2) representa la diferencia entre los coeficientes de acoplo de propagación de energía de la rama superior e inferior. Para un modulador MZ ideal, $\varepsilon=0$, lo cual indica que la potencia se divide en partes iguales en las dos ramas. A su vez, los términos de variación de fase $\Delta \phi$ dependen de la tensión de polarización $\mathrm{V}_{(\mathrm{dc})}$ este valor se define como aquella tensión que aplicada sobre los electrodos del dispositivo provoca un cambio de fase de $180^{\circ}$ sobre la señal óptica que se propaga por la guía de onda como consecuencia del efecto electroóptico. Del mismo modo depende de $V_{R F}$, el cual representa el voltaje necesario en las entradas de RF (superior e inferior) para provocar un cambio de fase de $180^{\circ}$ entre los dos brazos del interferómetro. Si se asume un coeficiente de acoplo $\mathrm{A}_{1}=\mathrm{A}_{2}=0.5$, y $\alpha=2$ (para unas pérdidas de inserción típicas de un modulador MZ de $6 \mathrm{~dB}$ ), la ecuación (1) se puede expresar como

$E_{\text {out }}(t)=\left(\frac{1}{\sqrt{2}}\right) E_{\text {in }}(t) \frac{\left[\left(\frac{1}{\sqrt{2}}\right) e^{\left(j \Delta \varphi_{1}\right)}+\left(\frac{1}{\sqrt{2}}\right) e^{\left( \pm j \Delta \varphi_{2}\right)}\right]}{2}$

Simplificando se obtiene la expresión general del campo eléctrico a la salida del modulador MZ

$E_{\text {out }}(t)=\left(\frac{1}{4}\right) E_{\text {in }}(t)\left[e^{\left(j \Delta \varphi_{1}\right)}+e^{\left( \pm j \Delta \varphi_{2}\right)}\right]$

Finalmente, la expresión de una señal en un sistema radio sobre fibra se puede definir mediante

$V_{R F}(t)=c(t)+e(t) \cos \left(\omega_{e} t+\alpha_{e}\right)$
Donde $c(t)$ es cualquier señal en banda base con cierta velocidad binaria transportada en la portadora óptica y $e(t)$ es la señal de datos que modula la portadora de RF definida por $\left(\omega_{e}=2 \pi f_{e}\right)$ más una constante de fase $\alpha_{e}$. El índice de modulación (IM) se define a través de la relación entre la amplitud de la señal moduladora y la tensión de desplazamiento de fase $V_{\pi}$.

$I M_{\text {señal }}=\frac{V_{\text {señal }}}{V_{\pi}}$

\section{Resultados y discusión}

En esta sección se presentan diferentes configuraciones de sistemas que permiten la generación de señales RoF usando técnicas de combinación óptica. Para cada una de ellas se presenta el modelo analítico acompañado de resultados de modelamiento que muestran la calidad de las señales RoF generadas en función de la variación de los índices de modulación de la señal de banda base $c(t)$ y RF $e(t)$, las cuales se definen respectivamente por

$c(t)=A_{1} V_{B B}(t)+V_{d c B B}$

$e(t)=A_{2} V_{D R F}(t)$

La señal de banda base $c(t)$ está conformada por una señal de información de banda base $V_{B B}(t)$ de amplitud $A_{1}$ y un voltaje DC $V_{d c B B}$. Por otro lado, la señal de radio frecuencia $e(t)$ está conformada por una señal de datos $V_{D R F}(t)$, con amplitud $A_{2}$. En este trabajo la señal $e(t)$ tiene una tasa de transmisión de 2.5 Gbps con una portadora de $10 \mathrm{GHz}$ y fase de oscilación nula. La señal $c(t)$ utilizada tiene una tasa de transmisión de $625 \mathrm{Mbps}$. El esquema de generación de señales para sistemas RoF usando combinación eléctrica de banda base y radiofrecuencia se modeló usando el software de simulación de sistemas ópticos VPI TransmissionMaker, en el cual la función de campo obtenida para cada esquema se evaluó para diferentes valores de índice de modulación de banda base y de RF. La evaluación de la calidad de la señal generada se realiza mediante el factor de calidad $Q$, que se define como (Freude et al., 2012)

$Q=\frac{m_{1}-m_{0}}{\sigma_{1}+\sigma_{0}}$

Donde $m_{i}$ y $\sigma_{i}$ representan la potencia media y desviación típica de potencia respectivamente del bit $i$ en el instante de decisión en el receptor. La tasa de error de 
bit (BER) definida en función de $Q$ está dada por (Freude et al., 2012)

$$
B E R=\frac{1}{2} \operatorname{erfc}\left(\frac{Q}{\sqrt{2}}\right)
$$

En donde $\operatorname{erfc}$ es la función de error complementaria.

\section{Generación en brazo doble}

En este esquema la señal de banda base y radio frecuencia se introducen al modulador Mach Zehnder por sus brazos superior e inferior, respectivamente. La figura 3 muestra la configuración para dicho sistema.

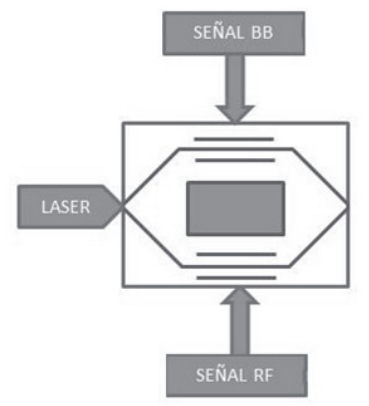

Figura 3. Esquema de modulación externa con dos brazos con MZ

Para este modelo la ecuación que describe el campo eléctrico a la salida es

$$
E_{\text {out }}(t)=\frac{E_{\text {in }}}{2} \cos \left(\frac{\Delta \varphi_{1}-\Delta \varphi_{2}}{2}\right) e^{\frac{j\left(\Delta \varphi_{1}+\Delta \varphi_{2}\right)}{2}}
$$

En donde la señal de banda base y de radiofrecuencia se introducen de manera independiente por cada uno de los brazos del modulador, (4) y (5) se reescriben en (15) y (16).

$$
\begin{aligned}
& \Delta \varphi_{1}=\frac{\pi}{V_{\pi(R F)}}\left[A_{1} V_{B B}(t)+V_{d c B B}\right]+\frac{\pi}{V_{\pi(d c)}} V_{1(d c)} \\
& \Delta \varphi_{2}=-\left\{\frac{\pi}{V_{\pi(R F)}} A_{2} V_{D R F}(t) \cos \left(\omega_{e} t+\phi_{e}\right)+\frac{\pi}{V_{\pi(d c)}} V_{2(d c)}\right\}
\end{aligned}
$$

Reemplazando (15) y (16) en (14) se obtiene la expresión (17) para el campo eléctrico de salida.

Nótese que en (17) se presenta un cambio de signo debido a un desfase de $180^{\circ}$ generado por la configuración interferométrica del modulador, la cual contribuye a la reducción del fenómeno de chirp. Finalmente se obtiene una expresión para el campo de salida en función de los índices de modulación de banda base y radiofrecuencia para el modelo de generación de doble brazo (ecuación 18).

El comportamiento del sistema en relación con el factor de calidad de las señales con variaciones en los índices de modulación de banda base y radiofrecuencia se observan en las figuras 4 y 5 . La figura 4 a muestra cómo un sistema de modulación de dos brazos genera una calidad adecuada para las señales de banda base con índices de modulación de radiofrecuencia $\left(m_{R F}\right)$ inferiores a 0.75 , en donde se obtiene un valor del factor de calidad $Q$ de aproximadamente 6.5 que equivale a un BER de $3.5 \times 10^{-11}$. Asimismo, se observa que la calidad de la señal de banda base mejora con el aumento de su propio valor de índice de modulación $\left(m_{B B}\right)$. Para valores de $m_{R F}$ superiores a 0.75 , la calidad de la señal de banda base disminuye debido a la menor disponibilidad de rango dinámico en el modulador. Por ejemplo, para $m_{R F}=0.9, Q_{B B} \approx 3.5$, lo cual equivale a un BER de $2.2 \times 10^{-4}$. Asimismo, la figura $4 \mathrm{~b}$ muestra el modelamiento de la calidad de la señal de RF en función de la variación de $m_{B B}$. Nuevamente se observa un desempeño adecuado para las señales de radiofrecuencia en ín-

$E_{\text {out }}(t)=\frac{E_{\text {in }}}{2} \cos \left(\frac{\pi}{2 V_{\pi(R F)}}\left[A_{1} V_{B B}(t)+V_{d c B B}+A_{2} V_{D R F}(t) \cos \left(\omega_{e} t+\phi_{e}\right)\right]+\frac{\pi}{2 V_{\pi(d c)}}\left[V_{1 d c}+V_{2(d c)}\right]\right) e^{\frac{j\left(\Delta \varphi_{1}+\Delta \varphi_{2}\right)}{2}}$

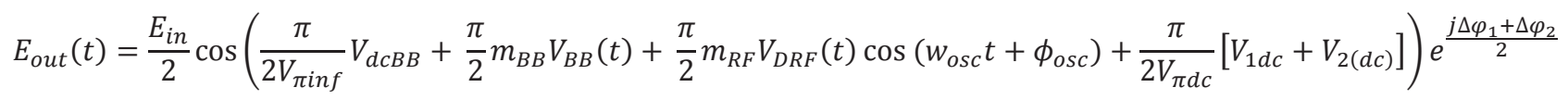




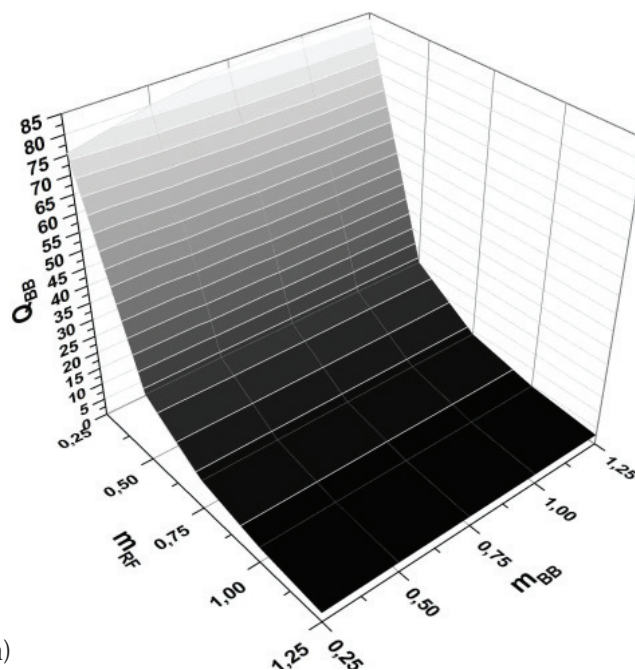

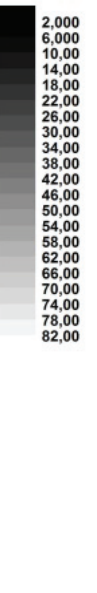

Figura 4. a) factor de calidad de la señal de banda base para generación con dos brazos, b) factor de calidad de la señal de RF para generación con dos brazos

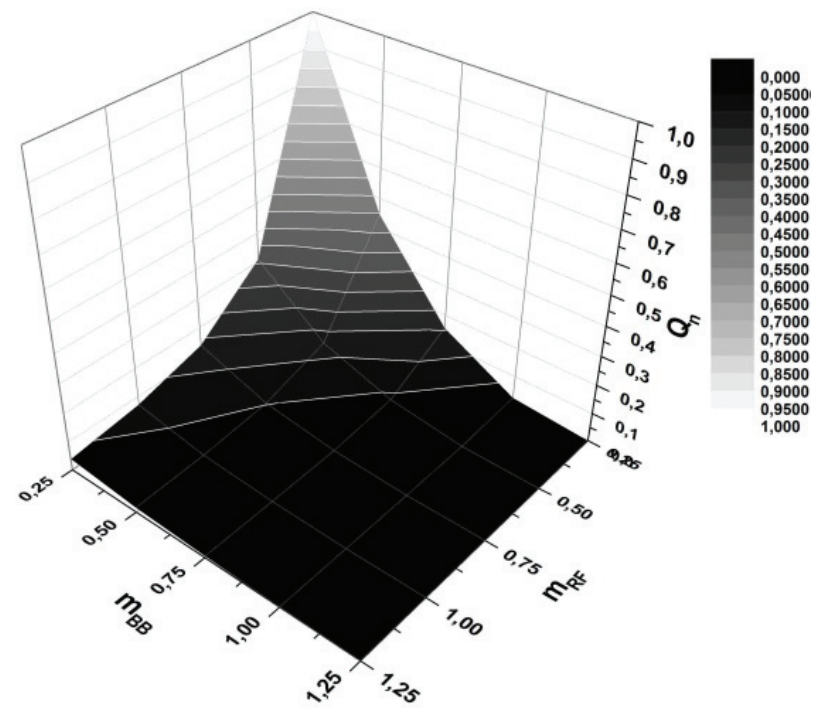

Figura 5. Figura de mérito para generación con dos brazos

dices de modulación de banda base inferiores a 0.75 , por ejemplo, para $m_{R F}=0.5, Q_{B B} \approx 8.5$, equivalente a un BER de $7.5 \times 10^{-18}$. Del mismo modo, si $m_{R F}=1, Q_{B B} \approx 2$ obteniéndose una tasa de error de $2.2 \times 10^{-2}$. Obsérvese también que la calidad de la señal de RF depende de su índice de modulación hasta cierto valor $\left(m_{R F}=1\right)$, a partir de ese punto la señal experimenta recorte debido a que la modulación se realiza en la zona menos lineal del modulador MZ.

La determinación del punto adecuado de trabajo se consigue a través de la figura de mérito, la cual representa el producto punto a punto de las figuras 4 y 5 . El resultado normalizado que se consigue se muestra en la figura 5, donde se observa que el punto óptimo de trabajo se obtiene para la combinación de los índices de modulación $m_{B B}=0.25$ y $m_{R F}=0.25$. Para valores de $m_{B B}$ entre 0.25 y 0.75 y $m_{R F}$ entre 0.25 y 0.5 se encuentran rangos de configuración en los cuales se consiguen factores de calidad $Q$ para señales de RF y banda base mayores o iguales a 6, área más clara de la figura 6, determinando los posibles puntos de operación del sistema en el cual se generan señales de banda base y RF con buena calidad. Fuera de este rango la recuperación de las señales es deficiente presentando factores de calidad $Q$ inferiores a 6.

\section{Generación en paralelo}

En este esquema tanto la señal de banda base como la señal de radiofrecuencia se modulan independientemente por cada Mach Zehnder para posteriormente multiplexarse mediante un acoplador óptico. El esquema para esta configuración se muestra en la figura 6 . Para este modelo las ecuaciones que describen los campos de salida se muestran a continuación

$$
\begin{aligned}
& E_{\text {out } 1}(t)=\frac{E_{\text {in }}}{\sqrt{2 L_{m z 1}}} \cos \left(\frac{\Delta \varphi_{1}}{2}\right) e^{\frac{j \Delta \varphi_{1}}{2}} \\
& E_{\text {out } 2}(t)=\frac{E_{\text {in }}}{\sqrt{2 L_{m z 2}}} \cos \left(\frac{\Delta \varphi_{2}}{2}\right) e^{\frac{j \Delta \varphi_{2}}{2}}
\end{aligned}
$$




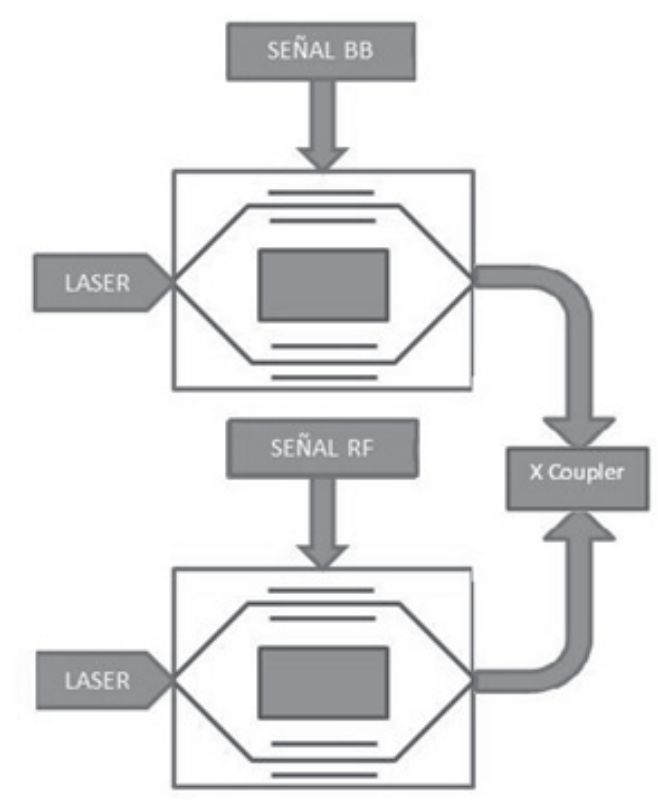

Figura 6. Esquema de modulación externa con dos moduladores $M Z$ en paralelo

$$
\begin{aligned}
& E_{\text {out } 1}(t)=\frac{E_{\text {in }}}{\sqrt{2 L_{m Z 1}}} \cos \left(\frac{\pi}{2 V_{\pi(R F M Z 1)}}\left[A_{1} V_{B B}(t)+V_{d c B B}\right]+\frac{\pi}{2 V_{\pi(d c M Z 1)}} V_{1 d c M Z 1}\right) e^{\frac{j \Delta \varphi_{1 M Z 1}}{2}} \\
& E_{\text {out } 2}(t)=\frac{E_{\text {in }}}{\sqrt{2 L_{m z 2}}} \cos \left(\frac{\pi}{2 V_{\pi(R F M Z 2)}} A_{2} V_{D R F}(t) \cos \left(\omega_{e} t+\phi_{e}\right)+\frac{\pi}{2 V_{\pi(d c M Z 2)}} V_{1 d c M Z 2}\right) e^{\frac{j \Delta \varphi_{1 M Z 2}}{2}}
\end{aligned}
$$

Donde $L_{m z}$ son las pérdidas del modulador. Puesto que la señal de banda base y radiofrecuencia se modulan de manera independiente en cada modulador $\mathrm{MZ}$, los campos de salida quedan definidos con lasecuaciones 21 y 22.

Donde el subíndice MZ1 y MZ2 corresponden al modulador 1 y 2 , respectivamente. Si se desea determinar un campo total de salida se utiliza un acoplador para obtener la suma de los campos de salida de ambos moduladores, que se describe por el siguiente sistema

$$
\left(\begin{array}{l}
E_{\text {outT } 1} \\
E_{\text {out } 2}
\end{array}\right)=\left(\begin{array}{cc}
\sqrt{1-\alpha} & j \sqrt{\alpha} \\
j \sqrt{\alpha} & \sqrt{1-\alpha}
\end{array}\right) *\left(\begin{array}{l}
E_{\text {out } 1} \\
E_{\text {out } 2}
\end{array}\right)
$$

A partir del acoplador se determina la siguiente expresión para el campo de salida

$$
E_{\text {out } 1}=\sqrt{1-\alpha} E_{\text {out } 1}+j \sqrt{\alpha} E_{\text {out } 2}
$$

Finalmente y considerando las mismas pérdidas en los dos moduladores y un valor en el coeficiente de acoplo $\alpha$ que garantice una distribución equitativa del campo, se obtiene la expresión (25) del campo eléctrico en función de los índices de modulación de banda base y radiofrecuencia.

Las figuras 7 y 8 muestran los resultados de modelado para este esquema. La figura 7a muestra el comportamiento de la calidad de la señal de banda base en función de $m_{R F}$. Se observa que la calidad de la señal de banda base tiene un comportamiento monótono creciente en función de su índice de modulación y que presenta buenos resultados para valores de $m_{R F}$ inferiores a 1. Por ejemplo, para $m_{R F}=0.5, Q_{B B}=8.6$ para $m_{B B}=0.25$, mientras que para el mismo valor de $m_{R F}$, con $m_{B B}=1$ se obtiene $Q_{B B} \approx 14$.6. Por otro lado, la figura $7 \mathrm{~b}$ muestra el modelamiento de la calidad de la señal de RF en función de $m_{B B}$. Los resultados muestran que las señales de

$E_{\text {out } T 1}=\frac{E_{\text {in }}}{4}\left\{\left[\cos \left(\frac{\pi}{2} m_{B B} V_{B B}(t)+\frac{\pi}{2 V_{\pi(R F M Z 1)}} V_{\text {dcBB }}+\frac{\pi}{2 V_{\pi d c M Z 1}} V_{1 d c M Z 1}\right) e^{\frac{j \Delta \varphi_{1 M Z 1}}{2}}\right]+j\left[\cos \left(\frac{\pi}{2} m_{R F} V_{D R F}(t) \cos \left(\omega_{e} t+\phi_{e}\right)+\frac{\pi}{2 V_{\pi d c M Z 2}} V_{1 d c M Z 2}\right) e^{\frac{j \Delta \varphi_{1 M Z 2}}{2}}\right]\right\}$ 


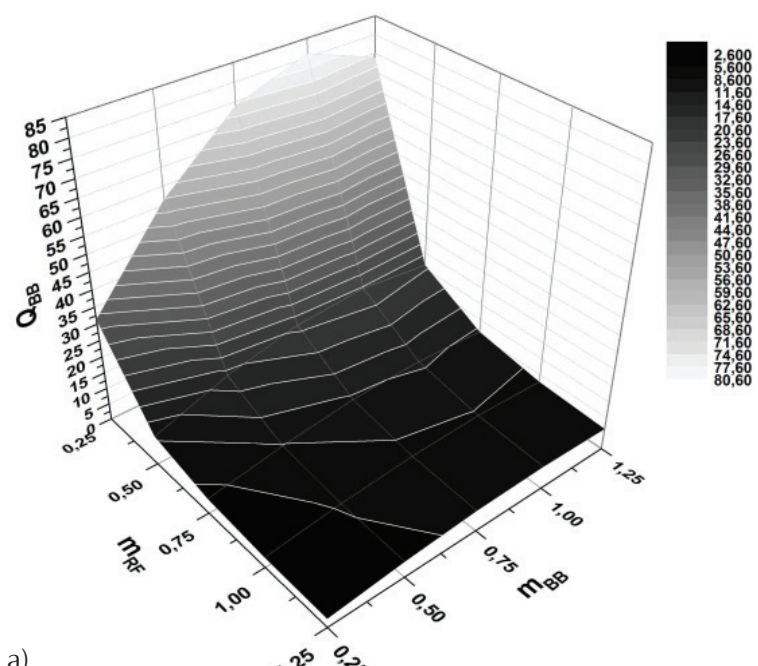

a)

Figura 7. a) factor de calidad de la señal de banda base para generación con dos $M Z$ en paralelo, b) factor de calidad de la señal de RF para generación con dos $\mathrm{MZ}$ en paralelo
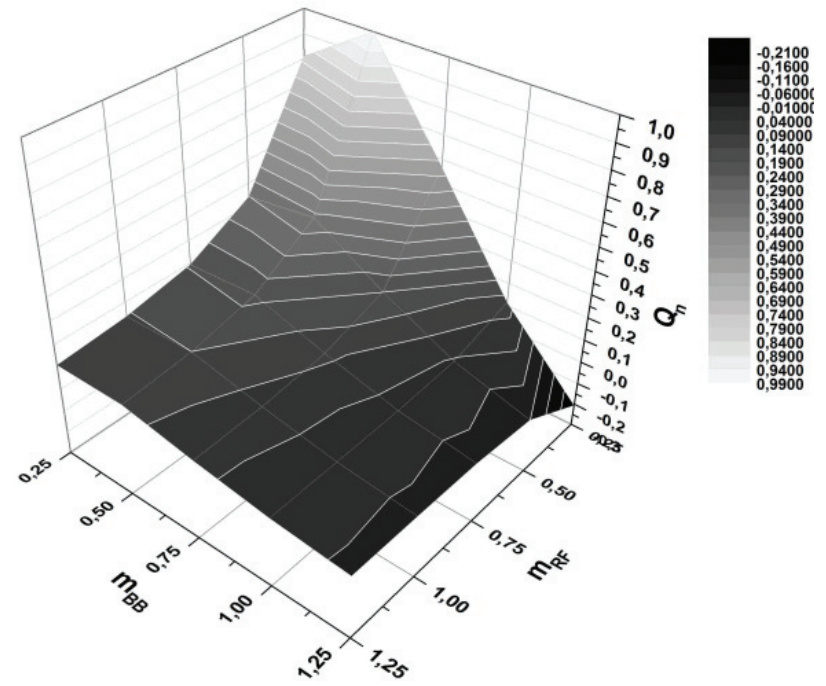

Figura 8. Figura de mérito para generación con dos MZ en paralelo

radiofrecuencia se generan con buena calidad en presencia de índices de modulación de banda base inferiores a 0.75 . El punto óptimo de trabajo se obtiene para la combinación de los índices de modulación $m_{B B}=0.5 \mathrm{y}$ $m_{R F}=0.25$. Para valores de $m_{B B}$ entre 0.5 y 1 y valores de $m_{R F}$ entre 0.25 y 0.5 , se encuentran rangos de configuración en los cuales se consiguen factores de calidad $Q$ para la señal de RF y banda base mayores o iguales a 6 obteniéndose tasas de error de $8.8 \times 10^{-10}$. Esta zona se representa en la parte más clara de la figura 8 . Al igual que en el sistema anterior, tal área determina los posibles puntos de operación del sistema en el cual se generan señales de banda base y RF con buena calidad.

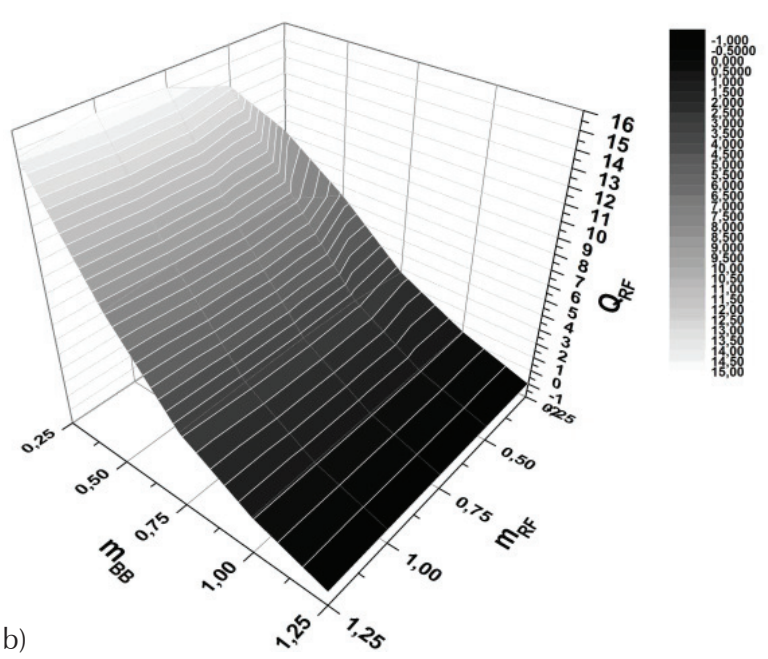

\section{Generación en serie}

En este esquema la señal de banda base y la señal de radiofrecuencia se modulan de manera independiente por dos Mach Zehnder que siguen una configuración en serie. La combinación es por lo tanto óptica, ya que la salida modulada del primer MZ actúa como entrada al segundo modulador. La figura 9 muestra la arquitectura de este esquema de generación.

Los campos de salida de cada uno de los moduladores MZ de este modelo se muestran a continuación

$$
\begin{aligned}
& E_{\text {outMZ1 }}(t)=\frac{E_{\text {in }}}{2} \cos \left(\frac{\Delta \varphi_{1 M Z 1}}{2}\right) e^{\frac{j \Delta \varphi_{1 M Z 1}}{2}} \\
& E_{\text {outMZ2 }}=\frac{E_{\text {out } M Z 1}(t)}{2} \cos \left(\frac{\Delta \varphi_{1 M Z 2}}{2}\right) e^{\frac{j \Delta \varphi_{1 M Z 2}}{2}}
\end{aligned}
$$

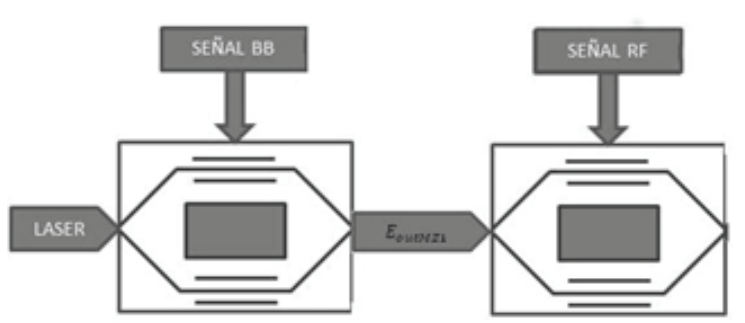

Figura 9. Esquema de modulación externa con dos moduladores MZ en serie 
Al igual que en el esquema de generación en paralelo, las señales de banda base y radiofrecuencia se introducen de manera independiente en cada modulador MZ, de este modo se obtiene el siguiente campo de salida para el primer modulador (Ecuación 28).

Puesto que el campo de entrada del segundo modulador corresponde al campo de salida del primer modulador, se obtiene la expresión de campo eléctrico en función de los índices de modulación de las señales de banda base y radiofrecuencia.

Los resultados obtenidos para el presente esquema se muestran en las figuras 10 y 11. Los resultados se basan en variaciones de índices de modulación de la señal de banda base entre 0.0833 y 0.25 , y de índices de modulación de radiofrecuencia con cambios iguales a los esquemas anteriores. Se selecciona un nivel de polarización de banda base de $1.25 \mathrm{~V}$ sobre el cual se ubica la señal. Los valores pequeños de índice de modulación de banda base reducen los problemas generados por la submodulación presente al combinar las señales de radiofrecuencia y banda base, nótese el doble coseno de la función de campo. Este aspecto impide recuperar adecuadamente la señal de radiofrecuencia. Como se puede observar en la figura 10a, el esquema de generación con dos moduladores MZ en serie ofrece buenos factores de calidad para la señal en banda base usando cualquier combinación de índices de modulación de RF y banda base. Se observa una tendencia monótona creciente con $m_{B B}$ y monótona decreciente con $m_{R F}$ en donde en el peor caso se consigue un factor de calidad $Q$ de 15 , es decir, un BER de $1.8 \times 10^{-51}$.

La figura $10 \mathrm{~b}$ representa el comportamiento del factor de calidad $Q$ de la señal $R F$, en función de los índices de modulación. Se observa que para cualquiera de las combinaciones de $m_{B B}$ y $m_{R F}$ se consigue un comportamiento adecuado a excepción de aquellas en las que $m_{B B} \geq 0.25$. Con valores iguales o superiores a 0.25 se genera una gran incidencia sobre la señal de radiofrecuencia debido al efecto de submodulación mencionado anteriormente. El punto óptimo de trabajo se obtiene para la combinación de los índices de modulación $m_{B B}=0.125$ y $m_{R F}=0.25$. Para valores de $m_{B B}$ entre 0 y 0.25 y valores de $m_{R F}$ entre 0.25 y 0.75 se encuentran rangos de configuración en los cuales se consiguen factores de calidad $Q$ superiores a 6 para las señales de RF y banda base. Esta zona se representa en la parte más clara de la figura 11. Las regiones de trabajo ineficientes las conforman las combinaciones restantes.

$$
\begin{aligned}
& E_{\text {outM } Z 1}(t)=\frac{E_{\text {in }}}{2} \cos \left(\frac{\pi}{2 V_{\pi(R F M Z 1)}}\left[A_{1} V_{B B}(t)+V_{d c B B}\right]+\frac{\pi}{2 V_{\pi d c M Z 1}} V_{1 d c M Z 1}\right) e^{\frac{j \Delta \varphi_{M Z 1}}{2}} \\
& E_{\text {out } 2}(t)=\frac{E_{\text {in }}}{4} \cos \left(\frac{\pi}{2} m_{B B} V_{B B}(t)+\frac{\pi}{2 V_{\pi(R F M Z 1)}} V_{d c B B}+\frac{\pi}{2 V_{\pi d c M Z 1}} V_{1 d c M Z 1}\right) \cos \left(\frac{\pi}{2} m_{R F} V_{D R F}(t) \cos \left(\omega_{e} t+\phi_{e}\right)+\frac{\pi}{2 V_{\pi d c M Z 2}} V_{1 d c M Z 2}\right) e^{\frac{j\left(\Delta \varphi_{1 M Z 1}+\Delta \varphi_{1 M Z 2}\right)}{2}}
\end{aligned}
$$
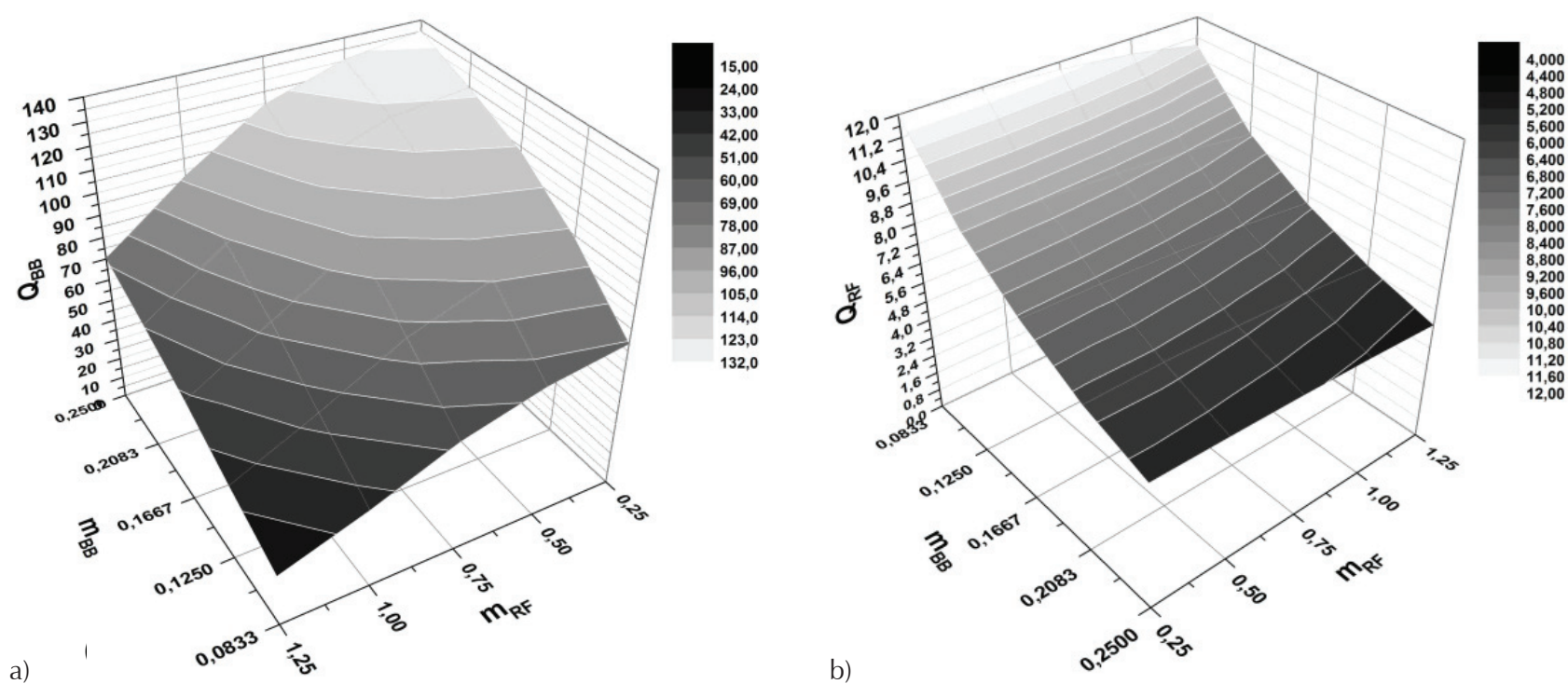

Figura 10. a) factor de calidad de la señal de banda base para generación con dos MZ en serie, b) factor de calidad de la señal de RF para generación con dos MZ en serie 


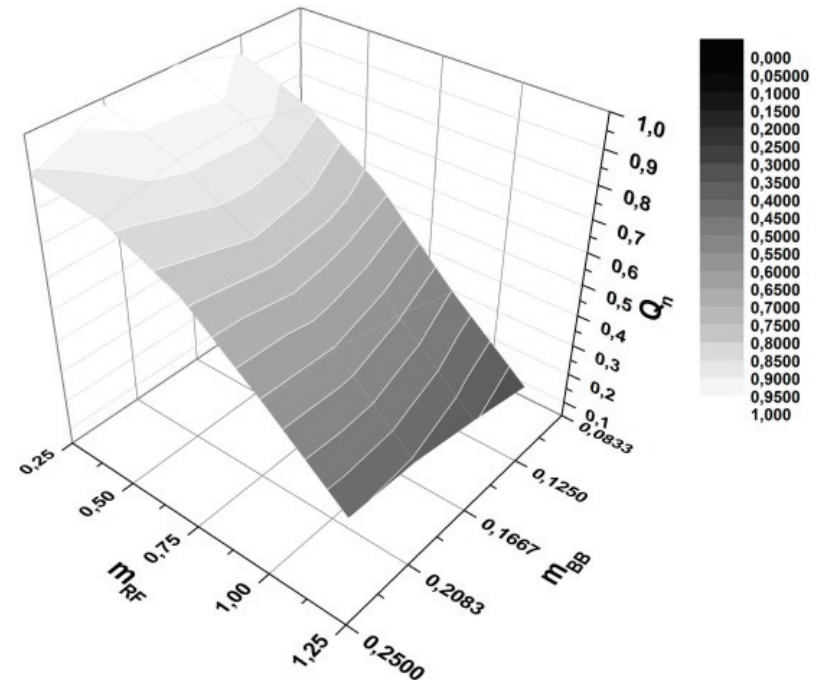

Figura 11. Figura de mérito para generación con dos $M Z$ en serie

\section{Análisis comparativo de los sistemas}

Con el fin de identificar el comportamiento general de los sistemas propuestos, las figuras 12 y 13 muestran una comparación de resultados en donde se reflejan los valores más altos del factor de calidad para la señal de banda base y para la señal de radiofrecuencia. Para el caso de la señal de banda base, la figura 12 muestra el factor de calidad $Q$ de dicha señal en función de $m_{B B}$ para un $m_{R F}$ fijo en 0.25 . Se observa en general, que el comportamiento del factor de calidad es monótono creciente con el valor del índice de modulación, como se observó anteriormente. Sin embargo, nótese como para $m_{B B}<1$ el esquema de generación de brazo doble mantiene valores casi constantes de $Q_{B B}$ si se compara con los valores obtenidos para el esquema de generación en paralelo. Este comportamiento se debe principalmente a la penalización en potencia que impone la combinación mediante el acoplador óptico, que afecta la relación de extinción de la señal de banda base para bajos valores de $m_{B B}$. Por otro lado, se observa que en el rango de $m_{B B}$ analizado para la generación serial, el comportamiento es creciente y la curva presenta una pendiente mayor que la obtenida con los otros esquemas, lo cual se debe a la independencia de uso del rango dinámico del modulador MZ.

En cuanto al comportamiento del factor de calidad de la señal de RF en función de , la figura 13 muestra los resultados obtenidos para los tres sistemas con fijo en 0.25. Nótese como los resultados para el esquema de brazo doble y generación en paralelo presentan una tendencia similar, no obstante, se observa nuevamente la penalización que sobre la relación de extinción de la señal de radiofrecuencia impone el sistema de combinación óptica. En cuanto al esquema serial, obsérvese la ligera degradación sufrida con el aumento de . Esto se debe a que a medida que aumenta, la excursión de señal en el modulador se va acercando a la zona menos lineal provocando recortes a dicha señal.

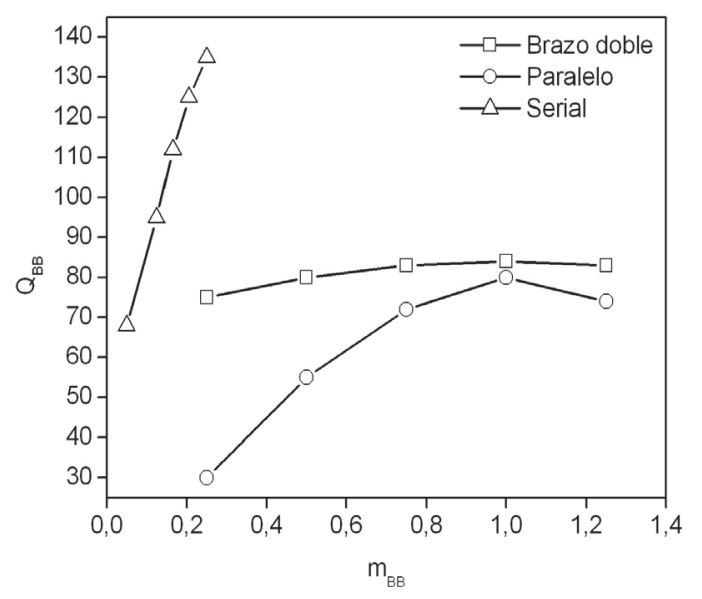

Figura 12. Resultados comparativos de $Q_{B B}$ para diferentes valores de $m_{B B}$ con $m_{R F}$ fijo en 0.25

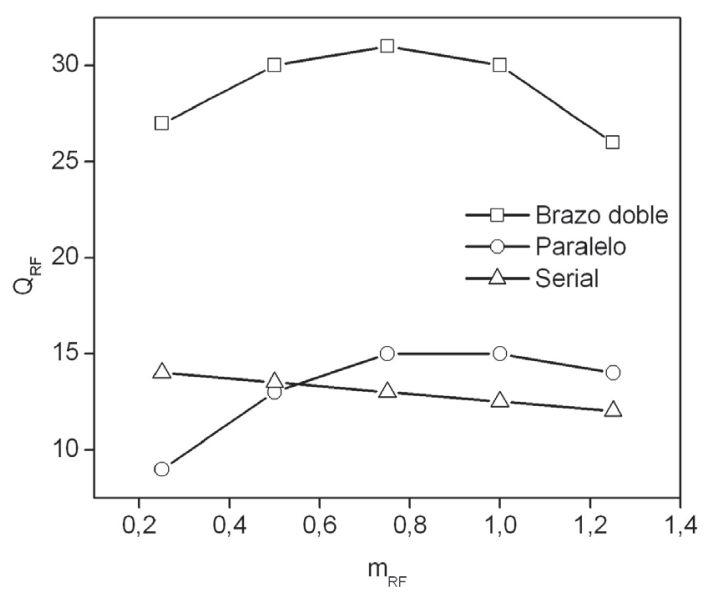

Figura 13. Resultados comparativos de $Q_{R F}$ para diferentes valores de $m_{R F}$ con $m_{B B}$ fijo en 0.25

\section{Conclusiones}

En todos los esquemas de modulación propuestos en el presente artículo es posible obtener una zona apropiada de trabajo para la trasmisión de señales de banda 
base y radiofrecuencia, y aunque estas regiones pueden parecer ineficientes dadas la pocas posibilidades de selección de índices adecuados de modulación dentro de la región lineal del modulador, se debe rescatar el hecho que en dichas franjas se pueden obtener factores de calidad buenos para cada una de las señales y que la determinación de dichas franjas permite prever, o al menos suponer, el esquema de mejor desempeño en relación con la calidad de las señales. El esquema de generación con dos moduladores $\mathrm{MZ}$ en paralelo, si bien presenta una mejora considerable de desempeño, bajo las mismas condiciones de trabajo de los sistemas de generación de dos brazos, también muestra un aumento de costos en el sistema, dada la necesidad de un modulador adicional. Se debe considerar una relación de costo-beneficio para las aplicaciones específicas donde se requiera un sistema de RoF con alto desempeño, que permitan determinar la pertinencia, o no, de un sistema con este tipo de configuración. Aunque el esquema de modulación con dos $\mathrm{MZ}$ en serie, parece tener una región de trabajo adecuada más amplia que en los otros esquemas, se debe hacer énfasis en el hecho que este se trabaja con índices de modulación de banda base pequeños y requiere una tensión de polarización que ubique la señal sobre la mitad superior de la zona lineal del modulador, de modo que el efecto sobre la señal de radiofrecuencia sea mínimo.

\section{Agradecimientos}

Los autores agradecen a la Universidad Distrital Francisco José de Caldas por el apoyo en la realización de este artículo.

\section{Referencias}

Ackerman E., Wanuga S., Kasemset D., Daryoush A., Samant N. Maximum dynamic range operation of a microwave external modulation fiber-optic link. IEEE Trans. Microw. Theory Tech, volumen 41 (número 8), agosto de 1993: 1299-1306.

Agrawal G. Fiber-Optic Communication Systems, 3a ed., John Wiley \& Sons, Nueva York, 2002.

Castleford D., Nirmalathas, Novak D., Tucker R. Optical crosstalk in fiber-radio WDM networks. IEEE Trans. Microw. Theory Tech, volumen 49 (número 10), octubre de 2001: 2030-2035.

Cho H.R., Shin M.J., Han S.H., Wu J.W. Mach-Zehnder interferometer measurement of the Pockels effect in a poled polymer film with a coplanar electrode structure. Applied Physics Lett, volumen 69 (número 25), octubre de 1996: 3788-3790.

Chun-Ting L., Jason C., Peng-Chun P., Cheng-Feng P., Wei-Ren P., Bi-Shiou C., Sien C. Hybrid Optical Access Network Integrating Fiber-to-the-Home and Radio-Over-Fiber Systems.
IEEE Photon Technol. Lett, volumen 19 (número 8), abril de 2007: 610-612.

Cisco, Cisco Visual Networking Index: Forecast and Methodology 2013-2018. Cisco [en línea] [fecha de consulta: 17 de julio de 2014]. Disponible en: http://www.cisco.com/en/US/solutions/ collateral/ns341/ns525/ns537/ns705/ns827/white_paper_c11481360.pdf.

Cox C. High-performance fiber-optic links for microwave applications, IEEE MTT-S International Microw., Symposium, Atlanta, junio, 1993.

Freude W., Schmogrow R., Nebendahl B., Winter M., Josten A., Hillerkuss D., Koenig S., Meyer J., Dreschmann M., Huebner M., Koos C., Becker J., Leuthold J. Quality metrics for optical signals: Eye diagram, Q-factor, OSNR, EVM and BER, 14th International Conference on Transparent Optical Networks (ICTON), Coventry, Inglaterra, julio, 2012.

Gee-Kung C., Jianjun Y., Zhensheng J. Architectures and enabling technologies for super-broadband radio-over-fiber opticalwireless access networks, IEEE International Topical Meeting on Microwave Photonics, Victoria, Canada, octubre, 2007.

Ho K. Generation of arbitrary quadrature signals using one dual drive modulator. IEEE J. Lightwave Technol, volumen 23 (número 2), febrero de 2005: 764-770.

Kaiser R., Velthaus K., Brast T., Gruner M., Hamacher M., Hoffmann D., Schell M. Medium and large scale Mach-Zehnder modulator ICs on InP for fabrication of advanced transmitters, en: International Conference on Transparent Optical Networks. Coventry, UK, julio, 2012.

Karim A., Devenport J. Noise figure reduction in externally modulated analog fiber-optic links. IEEE Photon. Technol. Lett, volumen 19 (número 5), marzo de 2007: 312-314.

Kikuchi N., Yamada E., Shibata Y., Ishii H. High-speed inp-based mach-zehnder modulator for advanced modulation formats, IEEE Compound Semiconductor Integrated Circuit Symposium, La Jolla, USA, octubre, 2012.

Kurniawan T., Nirmalathas A., Lim C., Novak D., Waterhouse R. Performance Analysis of Optimized Millimeter-Wave Fiber Radio Links. IEEE Trans. Microw. Theory Tech, volumen 54 (número 2), febrero de 2006: 921-928.

Lim C., Attygalle M., Nirmalathas A., Novak D., Waterhouse R. Analysis of optical carrier-to-sideband ratio for improving transmission performance in fiber-radio links. IEEE Trans. Microw. Theory Tech., volumen 54 (número 5), mayo de 2006: 2181-2187.

Marciniak M. Towards broadband global optical and wireless networking, $11^{\text {th }}$ Management Committee Meeting of COST Action 273, Towards Mobile Broadband Multimedia Networks, Duisburg, Germany, septiembre, 2004.

Mizuguti H., Okuno T., Komaki S., Morinaga N. Performance analysis of optical fiber link for microcellular mobile communication systems. IEICE Trans. Electron. E series, volumen 76 (número 2), febrero de 1993: 271-278. 
Prabu K., Bose S., Kumar D.S. Analysis of optical modulators for Radio over Free Space Optical Communication systems and Radio over Fiber systems, IEEE India Conference INDICON, Kochi, India, diciembre, 2012.

Sabella R. Performance analysis of wireless broadband systems employing optical fiber links. IEEE Trans. Commun., volumen 47 (número 5), mayo de 2001: 715-721.

Sabido D., Kazovsky L. Dynamic range of optically amplified RF optical links. IEEE Trans. Microw. Theory Tech., volumen 49 (número 10), octubre de 2001: 1950-1955.

Way W. Optical fiber-based microcellular systems: An overview. IEICE Trans. Commun., volumen 76 (número 9), septiembre de 1993: 1091-1102.

Yamada E., Shibata Y., Watanabe K., Yasui T., Ohki A., Mawatari H., Kanazawa S., Iga R., Ishii H. Demonstration of 50 Gbit/s 16QAM signal generation by novel 16QAM generation method using a dual-drive InP Mach-Zehnder modulator, en: Optical Fiber Communication Conference, Los Angeles, marzo, 2011.

Zhensheng J., Jianjun Y., Georgios E., Gee-Kung C. Key enabling technologies for optical-wireless networks: optical millimeter-wave generation, wavelength reuse, and architecture.
IEEE J. of Lightwave Technol., volumen 25 (número 11), noviembre de 2007: 3452-347.

Zongjie H., Zhang X., Shilie Z., Xiaofeng J., Hao C. Any bias point control of mach-zehnder electrooptic modulator and its applications in optimization of radio-over-fiber links, IEEE International Topical Meeting on Microwave Photonics, Singapur, octubre, 2011.

\section{Este artículo se cita:}

Citación estilo Chicago

Muñoz-Ortiz, Rafael Oswaldo, Manuel Arturo Cely-Mancipe, Gustavo Adolfo Puerto-Leguizamón, Carlos Arturo Suárez-Fajardo. Generación de señales para sistemas radio sobre fibra basados en combinación óptica. Ingeniería Investigación y Tecnología, XVI, 04 (2015): 585-598.

\section{Citación estilo ISO 690}

Muñoz-Ortiz R.O., Cely-Mancipe M.A., Puerto-Leguizamón G.A., Suárez-Fajardo C.A. Generación de señales para sistemas radio sobre fibra basados en combinación óptica. Ingeniería Investigación y Tecnología, volumen XVI (número 4), octubre-diciembre 2015: 585-598. 


\section{Semblanzas de los autores}

Rafael Oswaldo Muñoz-Ortiz. Es tecnólogo en mantenimiento electrónico e instrumentación (2008) e ingeniero electrónico (2014). Durante el pregrado inicia su labor en el Laboratorio de Ingeniería de Microondas, Electromagnetismo y Radiación, LIMER de la Universidad Distrital Francisco José de Caldas. En dicho grupo se une al proyecto de investigación: Dynamic architectures for converged optical access networks, donde desarrolló el trabajo de grado: "Generación de señales para sistemas de Radio Sobre Fibra por medio de modulación externa". Actualmente se desempeña como Core Network Engineer en Huawei Technologies Co. Ltd. Sus intereses de investigación incluyen sistemas de comunicaciones ópticas y de radiofrecuencia.

Manuel Arturo Cely-Mancipe. Tecnólogo en mantenimiento electrónico e instrumentación (2008) e ingeniero electrónico (2014). Durante el pregrado inicia su labor en el Laboratorio de Ingeniería de Microondas, Electromagnetismo y Radiación, LIMER de la Universidad Distrital Francisco José de Caldas. En dicho grupo se une al proyecto de investigación: Dynamic architectures for converged optical access networks, donde desarrolló el trabajo de grado: “Generación de señales para sistemas de Radio Sobre Fibra por medio de modulación externa". Sus intereses de investigación incluyen sistemas de comunicaciones ópticas y de radiofrecuencia.

Gustavo Adolfo Puerto-Leguizamón. Es ingeniero de telecomunicaciones. En 2003, se vinculó al grupo de comunicaciones ópticas y cuánticas de la Universidad Politécnica de Valencia. Es doctor en telecomunicaciones (2008) e investigador posdoctoral en el Instituto de Telecomunicaciones y Aplicaciones Multimedia de la misma universidad hasta 2011. Durante la etapa posdoctoral se desempeñó como co-líder del paquete de trabajo sobre nueva generación de tecnologías ópticas para red de acceso en el marco del proyecto europeo ALPHA (Architectures for Flexible Photonics Home and Access Networks). Fue director de investigación de ingeniería en la Universidad Sergio Arboleda y desde 2012 profesor asistente de la Universidad Distrital Francisco José de Caldas. A la fecha ha publicado más de 40 artículos en revistas y congresos internacionales en el campo de redes ópticas.

Carlos Arturo Suárez-Fajardo. Es ingeniero electrónico por la Universidad Distrital y licenciado en matemáticas por la universidad Pedagógica Nacional. Inició estudios doctorales en 2002 y se integró como investigador adscrito al grupo de radiación electromagnética (GRE) de la Universidad politécnica de Valencia (España), donde participó en tres proyectos de investigación relacionados con desarrollo de elementos radiantes y de agrupaciones de antenas y redes de conformación de haz, dos de ellos financiados por la comunidad europea y uno por el ministerio de ciencia y tecnología español. En 2003 obtuvo el título de especialista en telecomunicaciones, en 2004 el título de Magister en Telecomunicaciones y el de doctor en Telecomunicaciones en 2006 por la Universidad Politécnica de Valencia. En el año 1999 se vincula a la Facultad de Ingeniería de la Universidad Distrital Francisco José de Caldas. Es autor de más de 40 artículos en revistas indexadas y en congresos internacionales. 